\title{
Recent advances in the treatment of cystinosis
}

\author{
J.A. SCHNEIDER ${ }^{1 *}$, K.F. ClarK ${ }^{1}$, A.A. GREENE ${ }^{1}$, J.S. REISCH ${ }^{2}$, T.C. MARKELlo ${ }^{3,6}$, \\ W.A. GAHL ${ }^{3}$, J.G. THOENE ${ }^{4}$, P.K. NOONAN ${ }^{5}$ and K.A. BERRY ${ }^{5}$ \\ ${ }^{1}$ University of California San Diego, La Jolla, California; ${ }^{2}$ University of Texas \\ Southwestern Medical Center at Dallas, Dallas, Texas; ${ }^{3}$ National Institute of Child and \\ Human Development, Bethesda, Maryland: ${ }^{4}$ University of Michigan, Ann Arbor, \\ Michigan; ${ }^{5}$ Mylan Pharmaceuticals, Inc., Morgantown, West Virginia; ${ }^{6}$ Current address: \\ Medical College of Virginia, Richman, Virginia, USA. \\ *Correspondence: University of California San Diego, Division of Pediatric \\ Metabolism, Department of Pediatrics, 9500 Gilman Drive DEPT 0609 F, La Jolla, CA \\ 92093-0609, USA
}

\begin{abstract}
Summary: Cysteamine bitartrate capsules (Cystagon) have been approved by the US Food and Drug Administration for use in patients with nephropathic cystinosis. Plasma cysteamine concentrations were virtually identical at various times following ingestion of either cysteamine hydrochloride or Cystagon capsules in 24 normal control subjects. A transfer study was done with eight cystinosis patients who had been receiving either cysteamine hydrochloride or phosphocysteamine for many years. The plasma cysteamine concentration was significantly higher $2 \mathrm{~h}$ after Cystagon and the leukocyte cystine content was significantly lower at all times after Cystagon compared to older forms of the drug. These differences are probably the result of greater patient compliance in taking the capsules compared to the older, liquid forms of the drug. A new method for following the course of renal glomerular deterioration in diseases such as cystinosis has been published recently. This method was used to re-analyse data on the efficacy of cysteamine treatment and to re-analyse new data on treating cystinosis patients with either of two doses of cysteamine $\left(1.30 \mathrm{~g} / \mathrm{m}^{2}\right.$ per day and $1.95 \mathrm{~g} / \mathrm{m}^{2}$ per day $)$. This new method agrees well with other methods and shows that both doses of drug are equally effective in maintaining glomerular function.
\end{abstract}

This review of the treatment of cystinosis will be divided into three parts. First, we will describe our experience with cysteamine bitartrate capsules (Cystagon). This form of cysteamine was approved for treatment of nephropathic cystinosis by the US Food and Drug Administration on 15 August 1994. Next, we will discuss a new method to evaluate glomerular filtration rate over long periods in cystinosis patients. This method was reported in the August issue of Pediatric Nephrology (Manz and Gretz 1994). Finally, we will discuss practical aspects of treatment in this disease. 


\section{CYSTAGON (CYSTEAMINE BITARTRATE CAPSULES)}

The first form of cysteamine used to treat cystinosis was cysteamine hydrochloride (Thoene et al 1976). This is the least expensive form of the compound, but it has several disadvantages. In addition to being very foul smelling and tasting, it is extremely hygroscopic. Thus, for accurate dispensing, the entire amount received must be rapidly weighed and dissolved in water. Fortunately, its acid $\mathrm{pH}$ keeps the compound in its reduced state. We have found it to be stable for many years at room temperature. In the United States it has been formulated to contain $50 \mathrm{mg}$ cysteamine base $/ \mathrm{ml}$.

The next form of cysteamine used was the phosphorothioester of cysteamine, phosphocysteamine (Thoene and Lemons 1980). In its pure form it is odourless and tasteless. However, when manufactured in large enough quantity for clinical use it usually contains about $0.5 \%$ cysteamine. This is enough to make this form almost as unpleasant as cysteamine hydrochloride. Phosphocysteamine has generally been dispensed in packets of $12.2 \mathrm{~g}$. When dissolved in $100 \mathrm{ml}$ of water it contains $50 \mathrm{mg}$ cysteamine base $/ \mathrm{ml}$. Before phosphocysteamine was used in cystinosis patients it was tested and found to be equivalent to cysteamine hydrochloride (Smolin et al 1988). Phosphocysteamine is about three times as expensive as cysteamine hydrochloride and is difficult to manufacture in a reproducible way.

When Mylin Pharmaceuticals agreed to sponsor cysteamine for New Drug Approval for cystinosis they decided to use the bitartrate salt of this compound. This is a stable solid that is water-soluble. They manufacture two sizes of capsules, $50 \mathrm{mg}$ and $150 \mathrm{mg}$ of cysteamine base. In order to convince ourselves and the Food and Drug Administration that these capsules were equivalent to both cysteamine and phosphocysteamine, the capsules were tested in both normal control individuals and cystinosis patients.

Twenty-four healthy male control subjects were given either $1.05 \mathrm{~g}$ cysteamine hydrochloride in solution or $1.05 \mathrm{~g}$ cysteamine bitartate capsules (both expressed as $\mathrm{g}$ cysteamine base) in a random cross-over design with a 2-week 'wash-out' period between dose forms. The subjects fasted for 10h, had a standard breakfast at 07:30 and received the medication at 08:00. They had meals at noon and 18:00. Their plasma cysteamine concentrations following the drugs are shown in Figure 1. The mean peak cysteamine concentration is higher following the capsules and the curve is slightly shifted to the right. However, neither of these findings is statistically significant.

A 'transfer study' was done with eight cystinosis patients who had each received either cysteamine hydrochloride or phosphocysteamine for many years. The average age of these patients was 10 years. Each patient was changed to a dose of their usual drug as close as possible to $1.3 \mathrm{~g} / \mathrm{m}^{2}$ per day with increments of $50 \mathrm{mg} /$ dose. (When administered in liquid form the dose could be adjusted by $0.1 \mathrm{ml}(5 \mathrm{mg})$ increments. When administered as capsules the dose can only be changed by $50 \mathrm{mg}$ (one capsule) increments.) This allowed the patients to take the same dose of liquid medication that they would later take as capsules. The drug was given in four divided doses as close to every six hours as possible. On the fifth day the patients were admitted to a Clinical Research Center and continued on the same dose of cysteamine hydrochloride or phosphocysteamine. On both day 6 and day 7 they received these medications at 08:00 following a breakfast that was standardized for each patient according to their customary meal. Blood was obtained at baseline, 1, 2 and $6 \mathrm{~h}$ for measurement of plasma cysteamine and leukocyte cystine. The patients were then 


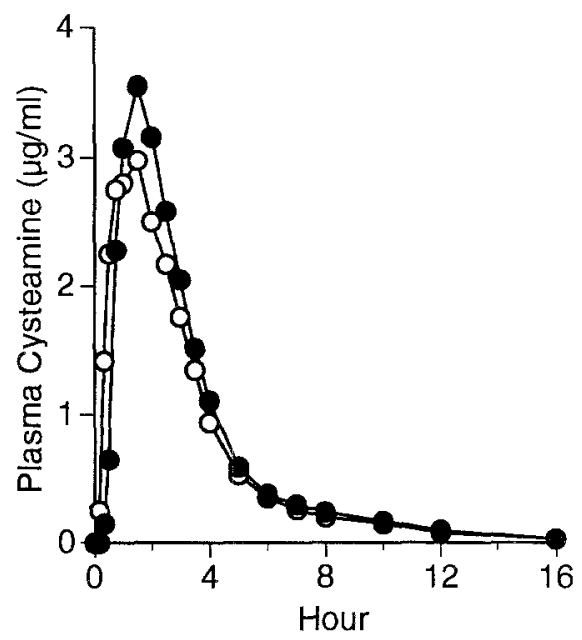

Figure 1 Plasma cysteamine concentration following either cysteamine hydrochloride solution (O) or cysteamine bitartrate capsules (-) in 24 normal control subjects. The values at $24 \mathrm{~h}$ were zero and are not plotted. See text for details

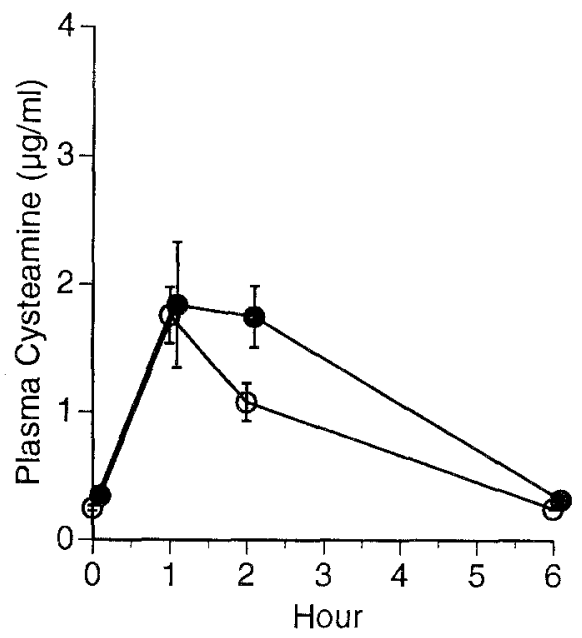

Figure 2 Plasma cysteamine concentration (mean \pm SEM) following either cysteamine hydrochloride or phosphocysteamine solution $(O)$ or cysteamine bitartrate capsules $(0)$ in eight cystinosis patients. A repeated measures analysis of variance was done with two repeated factors (form of drug and time) and comparisons of the dose forms at each time were done with Bonferroni adjustment of the $p$ values. At $2 \mathrm{~h}, p=0.006$. The symbols for the capsule values are plotted slightly to the right for clarity. See text for details

discharged from the hospital on the same dose of cysteamine, but as cysteamine bitartrate capsules. After three weeks they were readmitted for repeat studies, while receiving the capsules. The results are shown in Figures 2 and 3 .

The plasma cysteamine concentration was significantly higher at $2 \mathrm{~h}$ following the capsules as compared to the liquid forms of this medication (Figure 2). The reason for this 


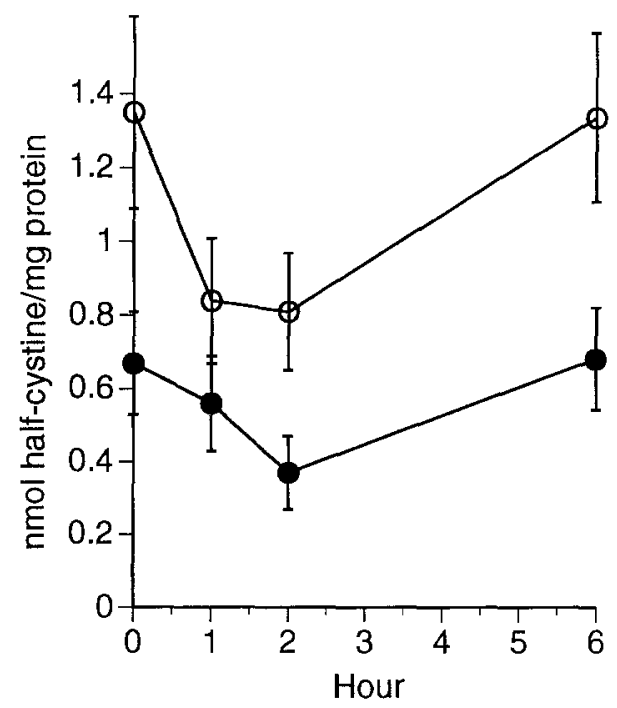

Figure 3 Leukocyte cystine content (mean \pm SEM) following either cysteamine hydrochloride or phosphocysteamine solution $(O)$ or cysteamine bitartrate capsules (-) in eight cystinosis patients. Statistical analysis was performed as in Figure 2. The repeated measures analysis of variance indicated that both the form of drug and time were significant factors. There was a differential response over time as indicated by the significant interaction $(p=0.007)$. The significance at each time point was $0 \mathrm{~h}, p=0.022 ; 1 \mathrm{~h}, p=0.067 ; 2 \mathrm{~h}, p=0.014 ; 6 \mathrm{~h}, p=0.026$. See text for details

is not clear. It may be related to the time required for the capsules to dissolve in the gut. However, this was not seen in the normal control subjects. Of greater surprise, the leukocyte cystine values were significantly lower following the capsules compared to the liquid forms of the drug (Figure 3). These values almost certainly reflect the amount of drug taken in the preceding several days. Thus, the differences are probably the result of greater patient compliance in taking the capsules compared to the liquid forms of the drug.

The US Food and Drug Administration approved cysteamine bitartrate capsules (Cystagon) on 15 August 1994. In the United States it will be distributed by Chronimed Pharmacy, 13911 Ridgedale Drive, Minnetonka, MN 55305; phone (800) 444-5951; fax (612) 541-4969.

\section{EVALUATION OF GFR IN A HISTORICAL GROUP OF CYSTINOSIS PATIENTS}

Evaluating new therapies for cystinosis has been a humbling experience for clinical investigators. In the past, both a low-sulphur diet (Crawhall et al 1968; Bickel et al 1973) and D-penicillamine (Clayton and Patrick 1961; Hambraeus and Broberger 1967; Crawhall et al 1968) were reported to be effective therapies for this disease, but were later found to be ineffective. The difficulty is that most centres see very few of these patients and so studies usually involve several medical centres. In addition, glomerular filtration rate is very difficult to measure accurately in infants and small children. Some medical centres have a special interest in this measurement; others do not. It is often difficult to obtain 


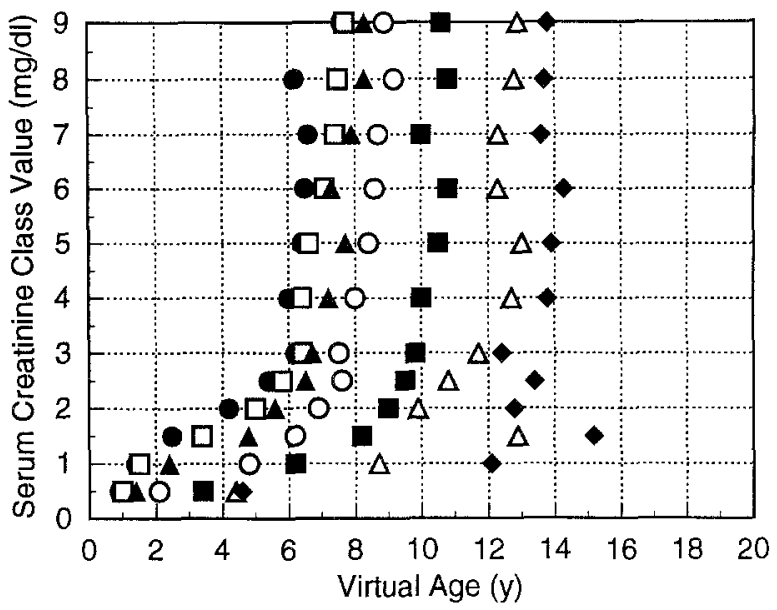

Figure 4 Standards for serum creatinine class values at each virtual age in patients with nephropathic cystinosis. These data are from 157 patients with both infantile and late-onset nephropathic cystinosis. 5th centile, $\mathbf{0} ; 10$ th, $\square ; 25$ th, $\mathbf{A} ; 50$ th, $\bigcirc 75$ th, $\mathbf{\square} ; 90 \mathrm{th}, \triangle ; 95 \mathrm{th}, \bullet$. See text for details. Redrawn from Manz and Gretz (1994)

reproducible results both within and between centres. Previous clinical studies in cystinosis have relied only on measurements of serum creatinine concentration and height by following the rise in serum creatinine concentration (Schneider et al 1979), calculated creatinine clearance (Gahl et al 1987), or predicted reciprocal serum creatinine at age 10 years (Gahl et al 1990). A recent study was especially important because it measured creatinine clearance based on 24-h urine collections in 76 cystinosis patients followed over a 32-year period at a single clinical research centre (Markello et al 1993).

A new method for following the course of renal glomerular deterioration in diseases such as cystinosis has been published recently (Manz and Gretz 1994). These authors took data from 205 cystinosis patients who had been followed at seven European centres before the availability of cysteamine (Gretz et al 1983). In brief, they first smoothed the longitudinal data of serum creatinine concentrations from each individual patient by the method of running medians. They next defined 12 classes of creatinine values $(<0.75 \mathrm{mg} / \mathrm{dl}=0.5 \mathrm{mg} / \mathrm{dl}, 0.75-1.24 \mathrm{mg} / \mathrm{dl}=1 \mathrm{mg} / \mathrm{dl}$, and so on $)$ and defined the term 'virtual age' as the '... age at the last smoothed serum creatinine value observed in one defined creatinine class within an uninterrupted chronological file of longitudinal smoothed serum creatinine values...'. This then allowed them to perform statistical analysis on the virtual ages for each creatinine class. (See Manz and Gretz (1994) for details.) They had adequate data to do these analyses on 157 patients and plotted the 5th, 10th, 25th, 50th, 75th, 90th and 95th centile for each creatinine class (Figure 4). They elected to include patients with late-onset cystinosis (Hooft et al 1971; Goldman et al 1971; Langman et al 1985) in these data and thus the curves are shifted to the right compared to what they would have been if all patients had infantile-onset nephropathic cystinosis. We have prepared a 'macro' in Panorama II for the Macintosh computer to evaluate data from our studies by this new method. (The macro is available without charge from J.A.S. You must have Panorama II in order to use it.) 


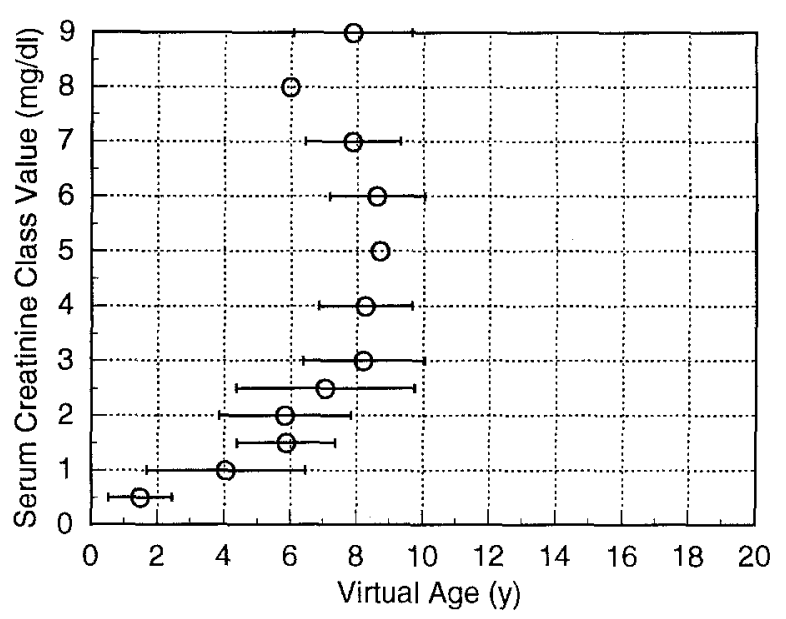

Figure 5 Virtual age (years) vs serum creatinine class (mg/dl) for patients who had never received cysteamine (Markello et al 1993). Virtual age was calculable for 26 of 27 such patients. Data were obtained from 351 visits. Mean \pm SD is plotted for each creatinine class unless there were fewer than three values in which case only the mean is shown

The study from the NIH Clinical Center (Markello et al 1993) included 27 patients who had never received cysteamine. Virtual age data could be calculated on 26 of theses patients as shown in Figure 5. The symbols in this figure show the mean \pm SD at each creatinine class. The 'curve' is very similar to the 50th centile in Figure 4. The NIH study also included 17 patients who were considered adequately treated with cysteamine. These adequately treated patients had started on cysteamine treatment before 2 years of age and had leukocyte cystine levels that averaged less than $2 \mathrm{nmol}$ half-cystine $/ \mathrm{mg}$ protein $5-6 \mathrm{~h}$ after receiving cysteamine. By the method in Manz and Gretz (1994), the virtual age is that at which a patient's serum creatinine concentration passes to the next creatinine class. Only 11 of the 17 adequately treated patients had creatinine values that ever increased enough to reach the next creatinine class. These data are shown in Figure 6. Figure 7 shows the data on all 17 patients, with the abscissa plotted as age rather than virtual age. These patients would appear to be at or above the 90 th centile of retention of glomerular function compared to the patients in Figure 4.

The first study with cysteamine in a cystinosis patient determined that $60 \mathrm{mg}$ cysteamine base per $\mathrm{kg}$ per day in four divided doses was a reasonable dose (Thoene et al 1976). This has continued to be the standard dose of cysteamine for these patients. With this treatment, patients maintained their glomerular function much longer and attained greater weight than in the past. For some patients a dose of $60 \mathrm{mg} / \mathrm{kg}$ became too great and we decided to base the dose on body surface area. We determined that a dose of $1.3 \mathrm{~g} / \mathrm{m}^{2}$ per day was equivalent to $60 \mathrm{mg} / \mathrm{kg}$ per day for the average-sized 4-year-old cystinosis patient and made this the standard dose. To determine whether a higher dose would be more beneficial, we compared the standard dose to one $50 \%$ higher $\left(1.95 \mathrm{~g} / \mathrm{m}^{2}\right.$ per day) (Clark et al 1992). Some of the results of this study are shown below.

Figures 8 and 9 show the creatinine data plotted according to Manz and Gretz (1994) for the patients receiving $1.3 \mathrm{~g} / \mathrm{m}^{2}$ per day. Virtual ages were calculable for only 48 of the 


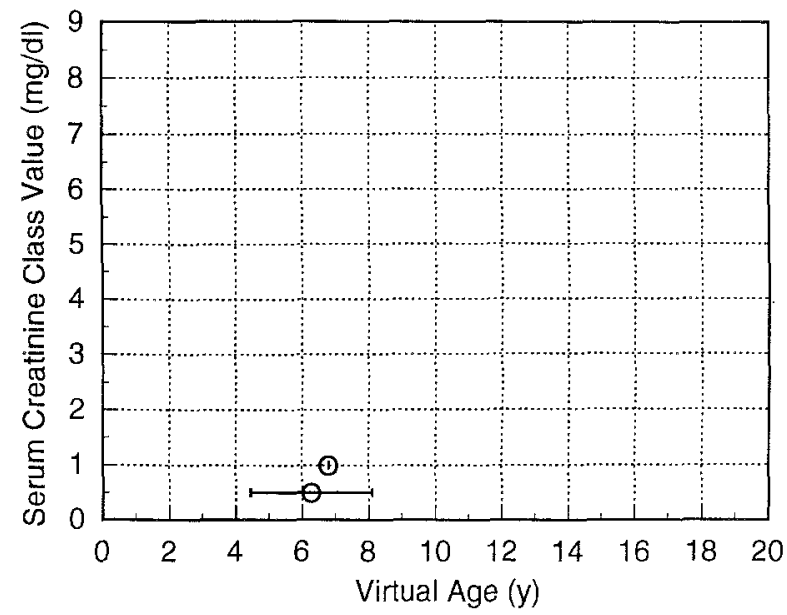

Figure 6 Virtual age (years) vs serum creatinine class (mg/dl) for patients who were considered to have received adequate treatment with cysteamine (Markello et al 1993). These patients began cysteamine before 2 years of age and maintained their mean leukocyte cystine value at below 2 nmol half-cystine/mg protein when blood was obtained 5-6h after medication. Virtual age was calculable for 11 of 17 such patients ( 7 at creatinine class 0.5 and 2 at creatinine class 1.0). Mean \pm SD is plotted for each creatinine class

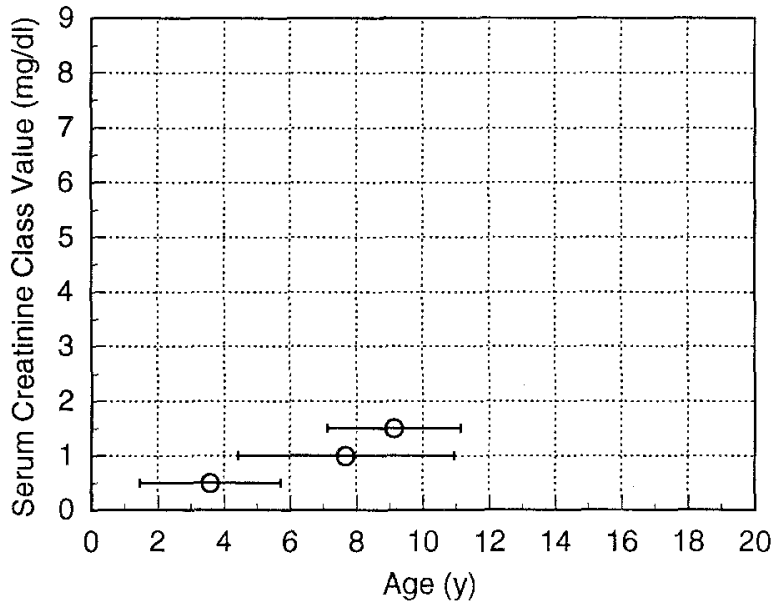

Figure 7 Mean $\pm S D$ is shown for the same patients as in Figure 6 except that the abscissa represents age (years) rather than virtual age. In this case data from 323 visits of all 17 patients are used

patients (Figure 8). Thus, we also plotted the data using age on the abscissa (Figure 9). This provided data from 901 visits in 90 patients. The curves are very similar in the two figures and are comparable to about the 90th centile in Figure 4. Figures 10 and 11 show the data plotted for the patients who received $1.95 \mathrm{~g} / \mathrm{m}^{2}$ per day. Figure 10 plots the virtual age on the abscissa and includes only 28 patients. By using age on the abscissa, the data from all 75 patients can be used (Figure 11). The curves are very similar and virtually identical to each other and to those from the patients on the lower dose. These data 


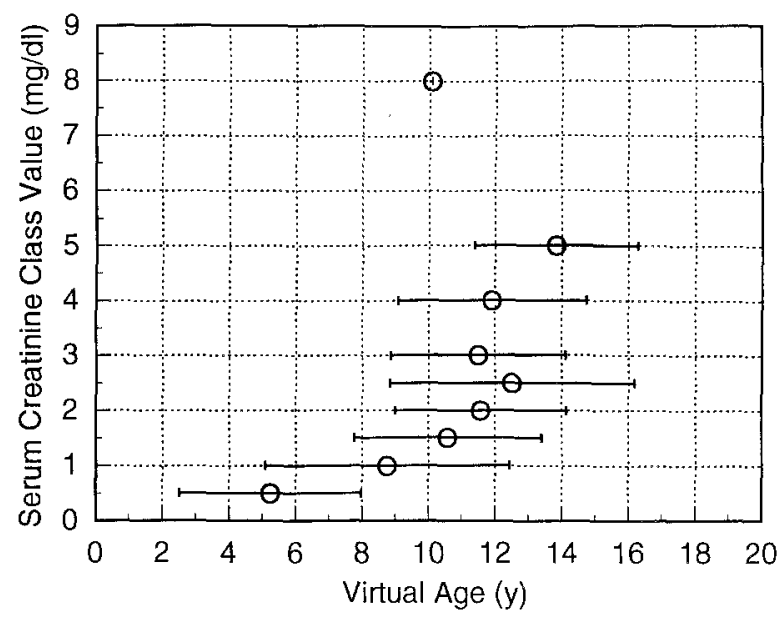

Figure 8 Virtual age (years) vs serum creatinine class ( $\mathrm{mg} / \mathrm{dl}$ ) for patients who received $1.3 \mathrm{~g} / \mathrm{m}^{2}$ per day of cysteamine. Mean \pm SD is plotted for each creatinine class except for the value at creatinine class $8 \mathrm{mg} / \mathrm{dl}$, which represents only one patient. Virtual age was calculable from 48 patients

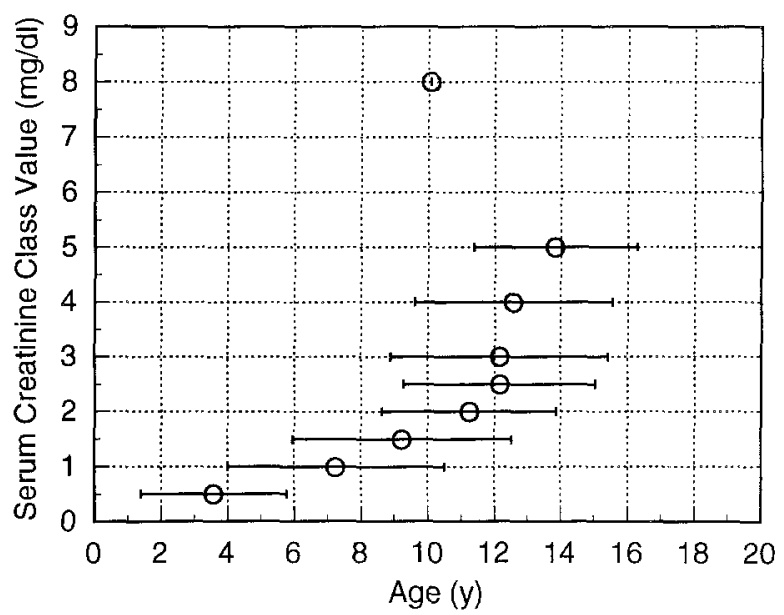

Figure 9 Data are shown for the same group of patients as in Figure 8 (dose of $1.3 \mathrm{~g} / \mathrm{m}^{2}$ per day of cysteamine) except that the abscissa represents age (years) rather than virtual age. Mean \pm SD is plotted for each creatinine class except for the value at creatinine class $8 \mathrm{mg} / \mathrm{dl}$, which represents only one patient. In this figure data from 901 visits of 90 patients are used

demonstrate the effectiveness of this drug in delaying glomerular deterioration in cystinosis patients. The reason more patients are shown for the lower than for the higher dose is that several patients could not tolerate the higher dose and exited the study. Most of these patients then received the lower dose, but were not included in the study.

The 165 patients whose data were plotted in Figures 8-11 include some who started the drug after they were 2 years of age and some who were not very compliant in taking the 


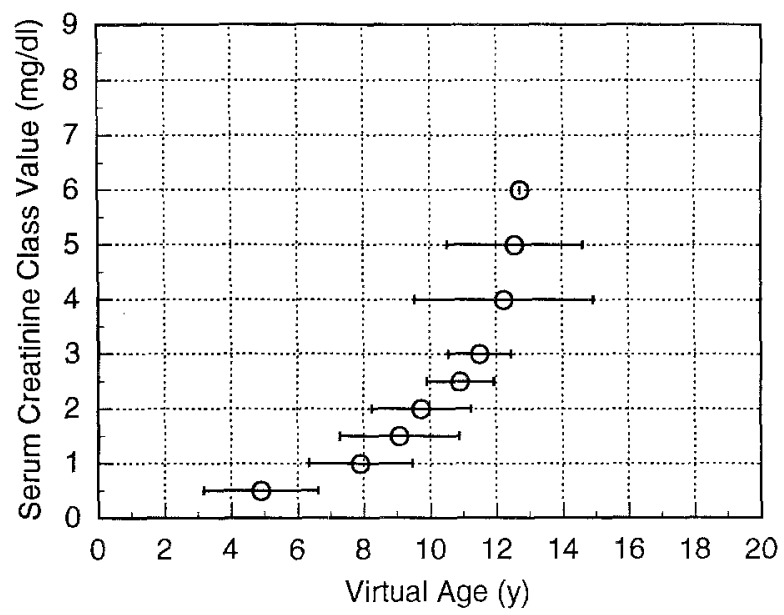

Figure 10 Virtual age (years) vs serum creatinine class ( $\mathrm{mg} / \mathrm{dl}$ ) for patients who received $1.95 \mathrm{~g} / \mathrm{m}^{2}$ per day of cysteamine. Mean $\pm S D$ is plotted for each creatinine class except for the value at creatinine class $6 \mathrm{mg} / \mathrm{dl}$, which represents only one patient. Virtual age was calculable for 28 patients

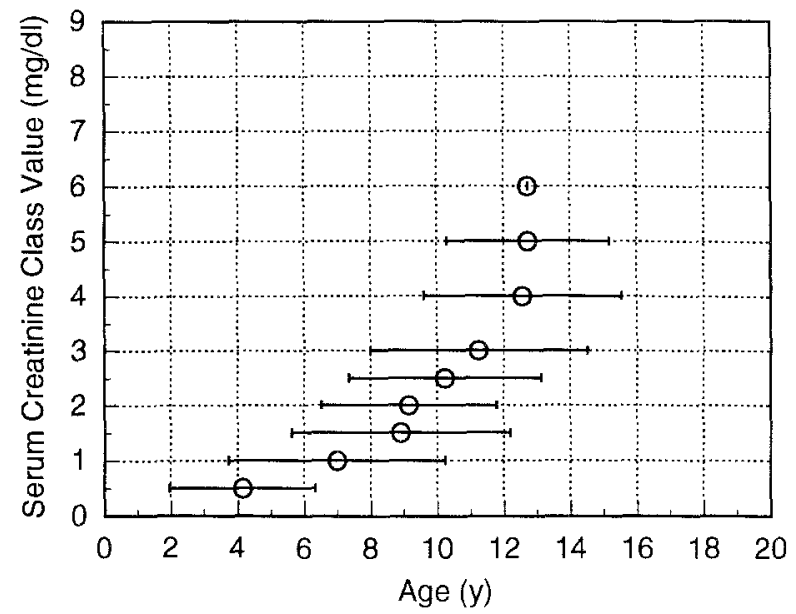

Figure 11 Data are shown for the same group of patients as in Figure 10 (dose of $1.95 \mathrm{~g} / \mathrm{m}^{2}$ per day of cysteamine) except that the abscissa represents age (years) rather than virtual age. Mean $\pm S D$ is plotted for each creatinine class except for the value at creatinine class $6 \mathrm{mg} / \mathrm{dl}$, which represents only one patient. In this figure data from 763 visits of 75 patients are used

drug. Still their average age of glomerular deterioration places them at or above the 90th centile for untreated cystinosis patients. (Remember that the data in Figure 4 include patients with late-onset cystinosis whose glomerular deterioration occurs at an older age.) The results in Figures $8-11$ are important for another reason. When the cysteamine hydrochloride and phosphocysteamine were given in solution $(50 \mathrm{mg} / \mathrm{ml})$, it was possible to change a patient's dose by increments of $5 \mathrm{mg}(0.1 \mathrm{ml})$. The smallest capsule of Cystagon is $50 \mathrm{mg}$. Thus, a patient's dose can be changed only by increments of $50 \mathrm{mg}$ 
Table 1 Approximate recommended dose of cysteamine for patients with nephropathic cystinosis

\begin{tabular}{lcc}
\hline $\mathrm{lb}$ & $\mathrm{kg}$ & mg per $6 \mathrm{~h}$ \\
\hline$<10$ & $<4.5$ & 100 \\
$10-20$ & $4.6-9.1$ & 150 \\
$20-30$ & $9.2-13.6$ & 200 \\
$30-40$ & $13.7-18.2$ & 250 \\
$40-50$ & $18.3-22.7$ & 300 \\
$50-70$ & $22.8-31.8$ & 350 \\
$70-90$ & $31.9-40.9$ & 400 \\
$90-110$ & $41.0-50.0$ & 450 \\
$>110$ & $>50.1$ & 500 \\
\hline
\end{tabular}

The dose should be checked each 3-4 months by obtaining blood 5-6h after receiving cysteamine and preparing leukocytes for cystine measurement. The optimum value is less than $1.0 \mathrm{nmol}$ half-cystine per $\mathrm{mg}$ protein

without opening capsules and dissolving them in liquid. The fact that the dose of cysteamine can range from 1.3 to $1.95 \mathrm{~g} / \mathrm{m}^{2}$ per day and still be effective in delaying glomerular deterioration is very reassuring.

\section{PRACTICAL SUGGESTIONS}

Participants at the 32nd Annual Symposium of the SSIEM in Edinburgh seemed especially interested in the following practical suggestions on the treatment of cystinosis with cysteamine.

(1) Start cysteamine therapy at as early an age as possible. It is now essential to determine whether patients with the Fanconi syndrome have cystinosis as an urgent diagnosis, so that cysteamine treatment can be started at once.

(2) Start new patients at a low dose and take 4-6 weeks to reach the 'full dose'.

(3) A good starting 'full dose' is $1.3 \mathrm{~g} / \mathrm{m}^{2}$ per day. Table 1 approximates this dose for most patients.

(4) Have the patient take this drug as close to every $6 \mathrm{~h}$ as possible. The drug is best tolerated if taken just after or with food.

(5) To ensure appropriate dosage and patient compliance, follow the patient's leukocyte cystine content every 3-4 months. Obtain the blood 5-6h after the medication is given for this measurement.

(6) Aim for a dose that keeps the leukocyte cystine below $1.0 \mathrm{nmol}$ half-cystine/mg protein. A value less than 2 is beneficial, but the patients who do best are those who are able to keep their level below 1 when the blood is obtained at the appropriate time.

Of course, there are several aspects of cystinosis treatment that are not related to cysteamine. There are many recent reviews on this topic (Schneider 1994; Gahl et al 1995).

\section{ACKNOWLEDGEMENT}

Support for these studies was provided by the Cystinosis Foundation, the Benard L. Maas Foundation, Contract HD-6-2927 from the National Institutes of Health, Grants M01-RR- 
00042 and M01-RR00827 from the General Clinical Research Centers Program of the National Center for Research Resources, National Institutes of Health, and Grants FD-R000215 and FD-U-00677 from the Food and Drug Administration Office of Orphan Products Development. In addition, support was provided from the Generic Pharmaceutical Industry Association Institute for Orphan Drugs, the Pharmaceutical Manufacturers Association, and Mylan Laboratories Inc.

\section{REFERENCES}

Bickel H, Lutz P, Schmidt H (1973) The treatment of cystinosis with diet or drugs. In Schulman JD, ed. Cystinosis Washington, DC: US Dept of Health, Education, and Welfare, No. (NIH) 72-249, $199-224$.

Clark KF, Franklin PS, Reisch JS, et al (1992) Effect of cysteamine- $\mathrm{HCl}$ and phosphocysteamine dosage on renal function and growth in children with nephropathic cystinosis. Clin Res 40: 113A (Abstract).

Clayton BE, Patrick AD (1961) Use of dimercaprol or pencillamine in the treatment of cystinosis. Lancet 2: 909.

Crawhall JC, Lietman PS, Schneider JA, Seegmiller JE (1968) Cystinosis. Plasma cystine and cysteine concentrations and the effect of D-pencillamine and dietary treatment. Am J Med 44: 330-339.

Gahl WA, Reed GF, Thoene JG, et al (1987) Cysteamine therapy for children with nephropathic cystinosis. $N$ Engl J Med 316: $971-977$.

Gahl WA, Schneider JA, Schulman JD, Thoene JG, Reed GF (1990) Predicted reciprocal serum creatinine at age 10 years as a measure of renal function in children with nephropathic cystinosis treated with oral cysteamine. Pediatr Nephrol 4: 129-135.

Gahl WA, Schneider JA, Aula PP (1995) Lysosomal transport disorders: cystinosis and sialic acid storage disorders. In Scriver CR, Beaudet AL, Sly WS, Valle D, eds. The Metabolic and Molecular Basis of Inherited Disease, 7th edn. New York: McGraw-Hill, 3763-3798.

Goldman H, Scriver CR, Aaron K, Delvin E, Canlas Z (1971) Adolescent cystinosis: comparisons with infantile and adult forms. Pediatrics 47: 979-988.

Gretz N, Manz F, Augustin R et al (1983) Survival time in cystinosis. A collaborative study. Proc Eur Dialysis Transplant Assoc 19: 582-589.

Hambraeus L, Broberger O (1967) Pencillamine treatment of cystinosis. Acta Paediatr Scand 56: $243-248$

Hooft C, Carton D, De Schrijver F, Delbeke MJ, Samijn W, Kint J (1971) Juvenile cystinosis in two siblings. In Carson NAJ, Raine DN, eds. Inherited Disorders of Sulphur Metabolism. Edinburgh and London: Churchill Livingstone, 141-149.

Langman CB, Moore ES, Thoene JG, Schneider JA (1985) Renal failure in a sibship with late-onset cystinosis. J Pediatr 107: 755-756.

Manz F, Gretz N (1994) Progression of chronic renal failure in a historical group of patients with nephropathic cystinosis. Pediatr Nephrol 8: 466-471.

Markello TC, Bernardini IM, Gahl WA (1993) Improved renal function in children with cystinosis treated with cysteamine. $N$ Engl J Med 328: $1157-1162$.

Schneider JA (1994) Cysteamine for treatment of cystinosis. In Andreucci VE, Fine LG, eds. International Yearbook of Nephrology Dialysis Transplantation, 9th edn. Oxford: Oxford University Press, 97-102.

Schneider JA, Schlesselman JJ, Mendoza SA, et al (1979) Ineffectiveness of ascorbic acid therapy in nephropathic cystinosis. $N$ Engl J Med 300: 756-759.

Smolin LA, Clark KF, Thoene JG, Gahl WA, Schneider JA (1988) A comparison of the effectiveness of cysteamine and phosphocysteamine in elevating plasma cysteamine concentration and decreasing leukocyte free cystine in nephropathic cystinosis. Pediatr Res 23: 616-20.

Thoene JG, Lemons R (1980) Cystine depletion of cystinotic tissues by phosphocysteamine (WR638). J Pediatr 96: 1043-1044.

Thoene JG, Oshima RG, Crawhall JC, Olson DL, Schneider JA (1976) Cystinosis. Intracellular cystine depletion by aminothiols in vitro and in vivo. J Clin Invest 58: 180-189. 